\section{Redshifts and distances}

IN the recent review by Burbidge ${ }^{1}$ I had expected to find a wide ranging discussion of all the important evidence both for and against cosmological origin of quasar redshifts. However, no mention is made of how the continuity of properties between galaxies and QSOs and amongst QSOs themselves supports the cosmological interpretation of all QSO redshifts, or how the number/redshift distribution of bright QSOs is found to be incompatible with non-cosmological redshifts ${ }^{2}$. Even within his rather restricted brief (dealing only with QSO/galaxy associations) Burbidge has failed to include results which do not favour his conclusions. He cites the existence of five 3C QSOs near bright galaxies as evidence for non-cosmological redshifts ${ }^{3}$. However, he does not mention several subsequent investigations ${ }^{4-6}$ of much larger samples of QSOs which failed to detect a statistically significant excess of close pairs compared with that expected by chance for a random distribution of quasars and galaxies.

Burbidge ignores published criticism of at least one of the arguments he uses. His Fig. 1 shows a correlation between galaxy redshift and separation of the galaxy from the associated QSO, and this is taken as good evidence for the galaxies and the QSOs being physically associated. Nevertheless, it has been pointed out by Noerdlinger ${ }^{7}$ and Browne and Cohen ${ }^{5}$ that when pairs selected from heterogeneous samples are used, strong selection effects inevitably give rise to a redshift-separation correlation roughly of the form presented by Burbidge. Note that not only is the legend to Fig. 1 confusing because it refers to a symbol not present in the diagram, but also the line shown is certainly not the best fit to all the points.

Finally, to establish the reality of noncosmological redshifts systematic investigations of well defined samples must be undertaken. Then, at least, it will be unnecessary to resort to estimating Arp's allocation of telescope time as a way of assessing the statistical significance of the results.

\section{IAN W. A. BROWNE}

Moss Cottage, Strawberry Lane, Wilmslow, Cheshire SK9 6AH, UK

Burbidge, (j. R. Nature 282, 451 (1979)

. Green. R. F \& Schmidt. M Astrophys. J. Lett. 220, L1 (1978).

Burbidge, E. M., Burbidge, G. R., Solomon, P. M. \& Strittmatter, P. A. Astrophys. J. 170, 233 (1971).

4. Hazard, C. \& Sanitt, N. Astrophys. Lett. 11, 77 (1972).

. Browne, I. W. A. \& Cohen, A. M. Mon. Not. R. astr. So 182, 181 (1978).

6. Nieto, J-L. Astr. Astrophys. 70, 219 (1978)

7. Noerdlinger. P. D. Astrophys. Space Sci. 38, 457 (1975).
BURBIDGE REPLIES - Browne is clearly strongly biased in favour of the cosmological redshift hypothesis, because none of his criticisms considers that I omitted discussion of the many problems associated with that hypothesis. His remarks imply that I have omitted only work critical of the evidence for noncosmological redshifts. In fact I attempted to demonstrate that there is good evidence for the existence of both cosmological and non-cosmological redshifts. I spent more time discussing the Arp evidence to attempt to redress the balance as the normal practice is to quote Stockton and ignore Arp.

In a previous review article ${ }^{1}$, which was far more wide-ranging, I did discuss all of the earlier evidence for association between galaxies and QSOs. In my later article I only quoted the 3C survey evidence for historical reasons, as I was concerned with an account of the more recent work. I had overlooked the paper of Browne and Cohen ${ }^{2}$ which should certainly be taken into account in evaluating my Fig. 1.

The results of Green and Schmidt ${ }^{3}$ lay outside my restricted brief. Had I discussed their results I would also have had to discuss other evidence, such as the existence of peaks and periodicities in the redshift distribution, the Compton problem, the Hubble diagram for QSOs, and the existence of so-called superlight velocities, all of which have to be discussed and evaluated before a balanced picture can be obtained.

\section{Geoffrey Burbidge}

Kitt Peak National Observatory, 950 North Cherry Avenue, PO Box 26732,

Tucson, Arizona 85726

1. Burbidge, G. Nature phys. Sci. 246, 17 (1973).

2. Browne, 1, W. A. \& Cohen, A. M. Mon. Not. R. ustr. Soc 182, $181(1978)$.

Green, R. F. \& Schmidt, M. Astrophys. J. Lett. 220, L (1978).

\section{The Lizard complex as an ophiolite}

KIRBY" has stated "Of prime importance to the proposed ophiolitic nature of the complex is the relationship between peridotite and gabbro...Green's lithological map and the author's field observations show that the gabbro is further restricted to the primary-type peridotite and does not occur in the recrystallized varieties except as isolated and deformed scraps".

This is an oversimplification: Green ${ }^{2}$ refers to the mineralogical assemblage within the primary peridotite and not to a completely unrecrystallized condition.
He wrote that the "coarse anhedral texture with curving grain boundaries" was igneous but concluded that "the primary peridotite assemblage has a...texture compatible with deep seated igneous crystallization or at least of crystallization in a hydrostatic or near hydrostatic stress environment. Although the banding and incipient augen foliation are ascribed to crystalline flow of the body at a very early stage, this flow has occurred at a rate accommodated in the rock without cataclasis or creation of granulitic or schistose fabrics".

All the evidence of Flett ${ }^{3}$, Green ${ }^{4}$ and Rothstein $^{5,6}$ taken together suggests that the rocks within the mass of primary peridotite south of Coverack have undergone a near solid-state deformation, although within this primary assemblage peridotite, in the lherzolites and harzburgites containing little or no troctolite, there are relics of primary textures and structures resembling crescumulates rather than settled gravity stratified cumulates. The deformation of the primary assemblage structures in this area is part of the continuity which exists between the two major areas of peridotite with respect to the trends of the near vertical foliation and banding, the primary mineralogy (to be reported elsewhere), and the rare earth content ${ }^{7}$. It would seem reasonable, therefore, to regard the peridotite as originally one body (albeit the eastern part not quite so heavily deformed as the western part). This being so the continuity extends between the two parts of the peridotite and the hornblende schists, and is broken, one way or the other, by the cross-cutting field relationships of the gabbro. This implies a contrary view to that expressed by Kirby" "that the association of peridotite and gabbro existed before their juxtapositioning with the surrounding hornblende schists (the event in which some of the peridotite was recrystallized)". This does not preclude the possibility of a deformed and recrystallized eastern part of the peridotite undergoing some partial melting.

\section{A. T. V. ROTHSTEIN}

Department of Geology,

Porstmouth Polytechnic,

Portsmouth, Hampshire PO1 3QL, UK

1. Kirby, G. A. Nature 282, $58-61$ (1979).

2. Green, D. H. I. Petrol. 5, 134-188 (1964)

. Flett, J. S. \& Hitl, J. B. Merm. geol. Sure, U.K. $(1912$, 1946).

4. Green, D. H. J. Genl. 72, 543--563 (1964)

5. Rothstein, A. T. V. Geol. Mag. 108, 39.3-398 (1971)

6. Rothstein, A. T. V. Proc. geol. Ass. 88, 93-105 (1977)

Frey, F. A. Geochim. cosmochim. Actl 33, 1429-1447 (1969).

KIRBY REPLIES The interrelationships between the foliations in the primary peridotite and those in the secondary peridotites and schists are not well 